\title{
Positive Solutions of Eigenvalue Problems for a Class of Fractional Differential Equations with Derivatives
}

\author{
Xinguang Zhang, ${ }^{1}$ Lishan Liu, ${ }^{2}$ \\ Benchawan Wiwatanapataphee, ${ }^{3}$ and Yonghong $\mathrm{Wu}^{4}$ \\ ${ }^{1}$ School of Mathematical and Informational Sciences, Yantai University, Yantai, Shandong 264005, China \\ ${ }^{2}$ School of Mathematical Sciences, Qufu Normal University, Qufu, Shandong 273165, China \\ ${ }^{3}$ Department of Mathematics, Faculty of Science, Mahidol University, Bangkok 10400, Thailand \\ ${ }^{4}$ Department of Mathematics and Statistics, Curtin University of Technology, Perth, WA 6845, Australia
}

Correspondence should be addressed to Xinguang Zhang, zxg123242@sohu.com

and Benchawan Wiwatanapataphee, scbww@mahidol.ac.th

Received 2 January 2012; Accepted 15 March 2012

Academic Editor: Shaoyong Lai

Copyright (C) 2012 Xinguang Zhang et al. This is an open access article distributed under the Creative Commons Attribution License, which permits unrestricted use, distribution, and reproduction in any medium, provided the original work is properly cited.

By establishing a maximal principle and constructing upper and lower solutions, the existence of positive solutions for the eigenvalue problem of a class of fractional differential equations is discussed. Some sufficient conditions for the existence of positive solutions are established.

\section{Introduction}

In this paper, we discuss the existence of positive solutions for the following eigenvalue problem of a class fractional differential equation with derivatives

$$
\begin{aligned}
-\boldsymbol{\Phi}_{\mathfrak{t}}{ }^{\alpha} x(t) & =\lambda f\left(t, x(t), \boldsymbol{\Phi}_{\mathbf{t}}{ }^{\beta} x(t)\right), \quad t \in(0,1), \\
\boldsymbol{\Phi}_{\mathfrak{t}}^{\beta} x(0)=0, & \boldsymbol{\Phi}_{\mathfrak{t}}^{\gamma} x(1)=\sum_{j=1}^{p-2} a_{j} \boldsymbol{\Phi}_{\mathfrak{t}}^{\gamma} x\left(\xi_{j}\right),
\end{aligned}
$$

where $\lambda$ is a parameter, $1<\alpha \leq 2, \alpha-\beta>1,0<\beta \leq \gamma<1,0<\xi_{1}<\xi_{2}<\cdots<\xi_{p-2}<1$, $a_{j} \in[0,+\infty)$ with $c=\sum_{j=1}^{p-2} a_{j} \xi_{j}^{\alpha-\gamma-1}<1$, and $\boldsymbol{\Phi}_{\mathbf{t}}$ is the standard Riemann-Liouville derivative. 
$f:(0,1) \times(0,+\infty) \times(0,+\infty) \rightarrow[0,+\infty)$ is continuous, and $f(t, u, v)$ may be singular at $u=0, v=0$, and $t=0,1$.

As fractional order derivatives and integrals have been widely used in mathematics, analytical chemistry, neuron modeling, and biological sciences [1-6], fractional differential equations have attracted great research interest in recent years [7-17]. Recently, ur Rehman and Khan [8] investigated the fractional order multipoint boundary value problem:

$$
\begin{aligned}
& \boldsymbol{\Phi}_{\mathbf{t}}^{\alpha} y(t)=f\left(t, y(t), \boldsymbol{\Phi}_{\mathbf{t}}^{\beta} y(t)\right), \quad t \in(0,1), \\
& y(0)=0, \quad \boldsymbol{\Phi}_{\mathbf{t}}^{\beta} y(1)-\sum_{i=1}^{m-2} \zeta_{i} \boldsymbol{\vartheta}_{\mathbf{t}}^{\beta} y\left(\xi_{i}\right)=y_{0}
\end{aligned}
$$

where $1<\alpha \leq 2,0<\beta<1,0<\xi_{i}<1, \zeta_{i} \in[0,+\infty)$ with $\sum_{i=1}^{m-2} \zeta_{i} \xi_{i}^{\alpha-\beta-1}<1$. The Schauder fixed point theorem and the contraction mapping principle are used to establish the existence and uniqueness of nontrivial solutions for the BVP (1.2) provided that the nonlinear function $f$ : $[0,1] \times \mathbb{R} \times \mathbb{R}$ is continuous and satisfies certain growth conditions. But up to now, multipoint boundary value problems for fractional differential equations like the BVP (1.1) have seldom been considered when $f(t, u, v)$ has singularity at $t=0$ and (or) 1 and also at $u=0, v=0$. We will discuss the problem in this paper.

The rest of the paper is organized as follows. In Section 2, we give some definitions and several lemmas. Suitable upper and lower solutions of the modified problems for the BVP (1.1) and some sufficient conditions for the existence of positive solutions are established in Section 3.

\section{Preliminaries and Lemmas}

For the convenience of the reader, we present here some definitions about fractional calculus.

Definition 2.1 (See $[1,6]$ ). Let $\alpha>0$ with $\alpha \in \mathbb{R}$. Suppose that $x:[a, \infty) \rightarrow \mathbb{R}$. Then the $\alpha$ th Riemann-Liouville fractional integral is defined by

$$
I^{\alpha} x(t)=\frac{1}{\Gamma(\alpha)} \int_{a}^{t}(t-s)^{\alpha-1} x(s) d s
$$

whenever the right-hand side is defined. Similarly, for $\alpha \in \mathbb{R}$ with $\alpha>0$, we define the $\alpha$ th Riemann-Liouville fractional derivative by

$$
\boldsymbol{\Phi}^{\alpha} x(t)=\frac{1}{\Gamma(n-\alpha)}\left(\frac{d}{d t}\right)^{(n)} \int_{a}^{t}(t-s)^{n-\alpha-1} x(s) d s
$$

where $n \in \mathbb{N}$ is the unique positive integer satisfying $n-1 \leq \alpha<n$ and $t>a$.

Remark 2.2. If $x, y:(0,+\infty) \rightarrow \mathbb{R}$ with order $\alpha>0$, then

$$
\boldsymbol{\Phi}_{\mathrm{t}}^{\alpha}(x(t)+y(t))=\boldsymbol{\Phi}_{\mathrm{t}}^{\alpha} x(t)+\boldsymbol{\Phi}_{\mathrm{t}}^{\alpha} y(t) .
$$


Lemma 2.3 (See [6]). One has the following.

(1) If $x \in L^{1}(0,1), v>\sigma>0$, then

$$
I^{v} I^{\sigma} x(t)=I^{v+\sigma} x(t), \quad \boldsymbol{\Phi}_{\mathrm{t}}^{\sigma} I^{v} x(t)=I^{v-\sigma} x(t), \quad \boldsymbol{\Phi}_{\mathrm{t}}{ }^{\sigma} I^{\sigma} x(t)=x(t) .
$$

(2) If $v>0, \sigma>0$, then

$$
\boldsymbol{\vartheta}_{\mathrm{t}}{ }^{\sigma} t^{\sigma-1}=\frac{\Gamma(\sigma)}{\Gamma(\sigma-v)} t^{\sigma-v-1}
$$

Lemma 2.4 (See [6]). Let $\alpha>0$. Assume that $x \in C(0,1) \cap L^{1}(0,1)$. Then

$$
I^{\alpha} \boldsymbol{\Theta}_{\mathrm{t}}^{\alpha} x(t)=x(t)+c_{1} t^{\alpha-1}+c_{2} t^{\alpha-2}+\cdots+c_{n} t^{\alpha-n},
$$

where $c_{i} \in \mathbb{R}(i=1,2, \ldots, n)$, and $n$ is the smallest integer greater than or equal to $\alpha$.

Let

$$
\begin{aligned}
& k_{1}(t, s)= \begin{cases}\frac{t^{\alpha-\beta-1}(1-s)^{\alpha-\gamma-1}-(t-s)^{\alpha-\beta-1}}{\Gamma(\alpha-\beta)}, & 0 \leq s \leq t \leq 1, \\
\frac{t^{\alpha-\beta-1}(1-s)^{\alpha-\gamma-1}}{\Gamma(\alpha-\beta)}, & 0 \leq t \leq s \leq 1,\end{cases} \\
& k_{2}(t, s)= \begin{cases}\frac{(t(1-s))^{\alpha-\gamma-1}-(t-s)^{\alpha-\gamma-1}}{\Gamma(\alpha-\beta)}, & 0 \leq s \leq t \leq 1, \\
\frac{(t(1-s))^{\alpha-\gamma-1}}{\Gamma(\alpha-\beta)}, & 0 \leq t \leq s \leq 1,\end{cases}
\end{aligned}
$$

and for $t, s \in[0,1]$, we have

$$
k_{i}(t, s) \leq \frac{(1-s)^{\alpha-\gamma-1}}{\Gamma(\alpha-\beta)}, \quad i=1,2
$$

Lemma 2.5. Let $h \in C(0,1)$; If $1<\alpha-\beta \leq 2$, then the unique solution of the linear problem

$$
\begin{gathered}
-\boldsymbol{\Phi}_{\mathfrak{t}}{ }^{\alpha-\beta} y(t)=h(t), \quad t \in(0,1), \\
y(0)=0, \quad \boldsymbol{\Phi}_{\mathbf{t}}^{\gamma-\beta} y(1)=\sum_{j=1}^{p-2} a_{j} \boldsymbol{\Phi}_{\mathfrak{t}}^{\gamma-\beta} y\left(\xi_{j}\right)
\end{gathered}
$$

is given by

$$
y(t)=\int_{0}^{1} K(t, s) h(s) d s,
$$


where

$$
K(t, s)=k_{1}(t, s)+\frac{t^{\alpha-\beta-1}}{1-\sum_{j=1}^{p-2} a_{j} \xi_{j}^{\alpha-\gamma-1}} \sum_{j=1}^{p-2} a_{j} k_{2}\left(\xi_{j}, s\right)
$$

is the Green function of the boundary value problem (2.9).

Proof. Applying Lemma 2.4, we reduce (2.9) to an equivalent equation:

$$
y(t)=-I^{\alpha-\beta} h(t)+c_{1} t^{\alpha-\beta-1}+c_{2} t^{\alpha-\beta-2}, \quad c_{1}, c_{2} \in \mathbb{R} .
$$

From (2.12) and noting that $y(0)=0$, we have $c_{2}=0$. Consequently the general solution of (2.9) is

$$
y(t)=-I^{\alpha-\beta} h(t)+c_{1} t^{\alpha-\beta-1} .
$$

Using (2.13) and Lemma 2.3, we have

$$
\begin{aligned}
\boldsymbol{\Phi}_{\mathfrak{t}}^{\gamma-\beta} y(t) & =-\boldsymbol{\Phi}_{\mathbf{t}}^{\gamma-\beta} I^{\alpha-\beta} h(t)+c_{1} \boldsymbol{\Phi}_{\mathfrak{t}}^{\gamma-\beta} t^{\alpha-\beta-1} \\
& =-I^{\alpha-\gamma} h(t)+c_{1} \frac{\Gamma(\alpha-\beta)}{\Gamma(\alpha-\gamma)} t^{\alpha-\gamma-1} \\
& =-\int_{0}^{t} \frac{(t-s)^{\alpha-\gamma-1}}{\Gamma(\alpha-\gamma)} h(s) d s+c_{1} \frac{\Gamma(\alpha-\beta)}{\Gamma(\alpha-\gamma)} t^{\alpha-\gamma-1} .
\end{aligned}
$$

Thus,

$$
\boldsymbol{\Phi}_{\mathfrak{t}}^{\gamma-\beta} y(1)=-\int_{0}^{1} \frac{(1-s)^{\alpha-\gamma-1}}{\Gamma(\alpha-\gamma)} h(s) d s+c_{1} \frac{\Gamma(\alpha-\beta)}{\Gamma(\alpha-\gamma)}
$$

and for $j=1,2, \ldots, p-2$,

$$
\boldsymbol{\Phi}_{\mathbf{t}}^{\gamma-\beta} w\left(\xi_{j}\right)=-\int_{0}^{\xi_{j}} \frac{\left(\xi_{j}-s\right)^{\alpha-\gamma-1}}{\Gamma(\alpha-\gamma)} h(s) d s+c_{1} \frac{\Gamma(\alpha-\beta)}{\Gamma(\alpha-\gamma)} \xi_{j}^{\alpha-\gamma-1} .
$$

Using $\boldsymbol{\Phi}_{\mathbf{t}}^{\gamma-\beta} y(1)=\sum_{j=1}^{p-2} a_{j} \boldsymbol{\Phi}_{\mathbf{t}}^{\gamma-\beta} y\left(\xi_{j}\right),(2.15)$, and (2.16), we obtain

$$
c_{1}=\frac{\int_{0}^{1}(1-s)^{\alpha-\gamma-1} h(s) d s-\sum_{j=1}^{p-2} a_{j} \int_{0}^{\xi_{j}}\left(\xi_{j}-s\right)^{\alpha-\gamma-1} h(s) d s}{\Gamma(\alpha-\beta)\left(1-\sum_{j=1}^{p-2} a_{j} \xi_{j}^{\alpha-\gamma-1}\right)} .
$$


So the unique solution of the problem (2.9) is

$$
\begin{aligned}
y(t)= & -\int_{0}^{t} \frac{(t-s)^{\alpha-\beta-1}}{\Gamma(\alpha-\beta)} h(s) d s+\frac{t^{\alpha-\beta-1}}{1-\sum_{j=1}^{p-2} a_{j} \xi_{j}^{\alpha-\gamma-1}} \\
& \times\left\{\int_{0}^{1} \frac{(1-s)^{\alpha-\gamma-1}}{\Gamma(\alpha-\beta)} h(s) d s-\sum_{j=1}^{p-2} a_{j} \int_{0}^{\xi_{j}} \frac{\left(\xi_{j}-s\right)^{\alpha-\gamma-1}}{\Gamma(\alpha-\beta)} h(s) d s\right\} \\
= & -\int_{0}^{t} \frac{(t-s)^{\alpha-\beta-1}}{\Gamma(\alpha-\beta)} h(s) d s+\int_{0}^{1} \frac{(1-s)^{\alpha-\gamma-1} t^{\alpha-\beta-1}}{\Gamma(\alpha-\beta)} h(s) d s \\
& +\frac{t^{\alpha-\beta-1}}{1-\sum_{j=1}^{p-2} a_{j} \xi_{j}^{\alpha-\gamma-1}} \sum_{j=1}^{p-2} a_{j} \int_{0}^{1} \frac{(1-s)^{\alpha-\gamma-1} \xi_{j}^{\alpha-\gamma-1}}{\Gamma(\alpha-\beta)} h(s) d s \\
& -\frac{t^{\alpha-\beta-1}}{1-\sum_{j=1}^{p-2} a_{j} \xi_{j}^{\alpha-\gamma-1}} \sum_{j=1}^{p-2} a_{j} \int_{0}^{\xi_{j}} \frac{\left(\xi_{j}-s\right)^{\alpha-\gamma-1}}{\Gamma(\alpha-\beta)} h(s) d s \\
= & \int_{0}^{1}\left(k_{1}(t, s)+\frac{t^{\alpha-\beta-1}}{1-\sum_{j=1}^{p-2} a_{j} \xi_{j}^{\alpha-\gamma-1}} \sum_{j=1}^{p-2} a_{j} k_{2}\left(\xi_{j}, s\right)\right) h(s) d s \\
= & \int_{0}^{1} K(t, s) h(s) d s .
\end{aligned}
$$

The proof is completed.

Lemma 2.6. The function $K(t, s)$ has the following properties.

(1) $K(t, s)>0$, for $t, s \in(0,1)$

(2) $t^{\alpha-\beta-1} \mathfrak{M}(s) \leq K(t, s) \leq M(1-s)^{\alpha-\gamma-1}$, for $t, s \in[0,1]$,

where

$$
\mathfrak{M}(s)=\frac{\sum_{j=1}^{p-2} a_{j} k_{2}\left(\xi_{j}, s\right)}{1-\sum_{j=1}^{p-2} a_{j} \xi_{j}^{\alpha-\gamma-1}}, \quad M=\frac{1+\sum_{j=1}^{p-2} a_{j}\left(1-\xi_{j}^{\alpha-\gamma-1}\right)}{\Gamma(\alpha-\beta)\left(1-\sum_{j=1}^{p-2} a_{j} \xi_{j}^{\alpha-\gamma-1}\right)}
$$

Proof. It is obvious that (1) holds.

From (2.11), we obtain

$$
K(t, s) \geq \frac{t^{\alpha-\beta-1}}{1-\sum_{j=1}^{p-2} a_{j} \xi_{j}^{\alpha-\gamma-1}} \sum_{j=1}^{p-2} a_{j} k_{2}\left(\xi_{j}, s\right)=t^{\alpha-\beta-1} \mathfrak{M}(s)
$$


From (2.8), we have

$$
\begin{aligned}
K(t, s) & =k_{1}(t, s)+\frac{t^{\alpha-\beta-1}}{1-\sum_{j=1}^{p-2} a_{j} \xi_{j}^{\alpha-\gamma-1}} \sum_{j=1}^{p-2} a_{j} k_{2}\left(\xi_{j}, s\right) \\
& \leq \frac{(1-s)^{\alpha-\gamma-1}}{\Gamma(\alpha-\beta)}+\frac{\sum_{j=1}^{p-2} a_{j}}{1-\sum_{j=1}^{p-2} a_{j} \xi_{j}^{\alpha-\gamma-1}} \frac{(1-s)^{\alpha-\gamma-1}}{\Gamma(\alpha-\beta)} \\
& \leq\left(1+\frac{\sum_{j=1}^{p-2} a_{j}}{1-\sum_{j=1}^{p-2} a_{j} \xi_{j}^{\alpha-\gamma-1}}\right) \frac{(1-s)^{\alpha-\gamma-1}}{\Gamma(\alpha-\beta)} .
\end{aligned}
$$

The proof is completed.

Consider the modified problem of the BVP (1.1):

$$
\begin{gathered}
-\boldsymbol{\Phi}_{\mathfrak{t}}{ }^{\alpha-\beta} y(t)=\lambda f\left(t, I^{\beta} y(t), y(t)\right), \\
y(0)=0, \quad \boldsymbol{\Phi}_{\mathfrak{t}}^{\gamma-\beta} y(1)=\sum_{j=1}^{p-2} a_{j} \boldsymbol{\Phi}_{\mathfrak{t}}^{\gamma-\beta} y\left(\xi_{j}\right) .
\end{gathered}
$$

Lemma 2.7. Let $x(t)=I^{\beta} y(t)$ and $y(t) \in C[0,1]$; then problem (1.1) is turned into (2.22). Moreover, if $y \in C([0,1],[0,+\infty))$ is a solution of problem (2.22), then the function $x(t)=I^{\beta} y(t)$ is a positive solution of the problem (1.1).

Proof. Substituting $x(t)=I^{\beta} y(t)$ into (1.1) and using Definition 2.1 and Lemmas 2.3 and 2.4, we obtain

$$
\begin{aligned}
\boldsymbol{\Phi}_{\mathrm{t}}^{\alpha} x(t) & =\frac{d^{n}}{d t^{n}} I^{n-\alpha} x(t)=\frac{d^{n}}{d t^{n}} I^{n-\alpha} I^{\beta} y(t) \\
& =\frac{d^{n}}{d t^{n}} I^{n-\alpha+\beta} y(t)=\boldsymbol{\Phi}_{\mathrm{t}}^{\alpha-\beta} y(t), \\
\boldsymbol{\Phi}_{\mathfrak{t}}^{\beta} x(t) & =\boldsymbol{\Phi}_{\mathrm{t}}^{\beta} I^{\beta} y(t)=y(t) .
\end{aligned}
$$

Consequently, $\boldsymbol{\Phi}_{\mathbf{t}}^{\beta} x(0)=y(0)=0$. It follows from $\boldsymbol{\Phi}_{\mathbf{t}}^{\gamma} x(t)=d^{n} / d \mathrm{t}^{n} I^{n-\gamma} x(t)=$ $\left(d^{n} / d t^{n}\right) I^{n-\gamma} I^{\beta} y(t)=\left(d^{n} / d t^{n}\right) I^{n-\gamma+\beta} y(t)=\boldsymbol{\Phi}_{\mathfrak{t}}^{\gamma-\beta} y(t)$ that $\boldsymbol{\Phi}_{\mathfrak{t}}^{\gamma-\beta} y(1)=\sum_{j=1}^{p-2} a_{j} \boldsymbol{\Phi}_{\mathfrak{t}}^{\gamma-\beta} y\left(\xi_{j}\right)$. Using $x(t)=I^{\beta} y(t), y \in C[0,1]$, we transform (1.1) into (2.22).

Now, let $y \in C([0,1],[0,+\infty))$ be a solution for problem (2.22). Using Lemma 2.3, (2.22), and (2.23), one has

$$
\begin{aligned}
-\boldsymbol{\Phi}_{\mathfrak{t}}{ }^{\alpha} x(t) & =-\frac{d^{n}}{d t^{n}} I^{n-\alpha} x(t)=-\frac{d^{n}}{d t^{n}} I^{n-\alpha} I^{\beta} y(t)=-\frac{d^{n}}{d t^{n}} I^{n-\alpha+\beta} y(t)=-\boldsymbol{\Phi}_{\mathfrak{t}}{ }^{\alpha-\beta} y(t) \\
& =\lambda f\left(t, I^{\beta} y(t), y(t)\right)=\lambda f\left(t, x(t), \boldsymbol{\Phi}_{\mathfrak{t}}{ }^{\beta} x(t)\right), \quad 0<t<1 .
\end{aligned}
$$


Noting

$$
\boldsymbol{\Phi}_{\mathbf{t}}^{\beta} x(t)=\boldsymbol{\Phi}_{\mathbf{t}}^{\beta} I^{\beta} y(t)=y(t), \quad \boldsymbol{\Phi}_{\mathbf{t}}^{\gamma} x(t)=\boldsymbol{\Phi}_{\mathbf{t}}^{\gamma-\beta} y(t),
$$

we have

$$
\boldsymbol{\Phi}_{\mathbf{t}}^{\beta} x(0)=0, \quad \boldsymbol{\Phi}_{\mathfrak{t}}^{\gamma} x(1)=\sum_{j=1}^{p-2} a_{j} \boldsymbol{\Phi}_{\mathfrak{t}}^{\gamma} x\left(\xi_{j}\right)
$$

It follows from the monotonicity and property of $I^{\beta}$ that

$$
I^{\beta} y \in C([0,1],[0,+\infty)) \text {. }
$$

Consequently, $x(t)=I^{\beta} y(t)$ is a positive solution of the problem (1.1).

Definition 2.8. A continuous function $\psi(t)$ is called a lower solution of the BVP (2.22), if it satisfies

$$
\begin{gathered}
-\boldsymbol{\vartheta}_{\mathfrak{t}}^{\alpha-\beta} \psi(t) \leq \lambda f\left(t, I^{\beta} \psi(t), \psi(t)\right), \\
\psi(0) \geq 0, \quad \boldsymbol{\vartheta}_{\mathfrak{t}}^{\gamma-\beta} \psi(1) \geq \sum_{j=1}^{p-2} a_{j} \boldsymbol{\vartheta}_{\mathfrak{t}}^{\gamma-\beta} \psi\left(\xi_{j}\right) .
\end{gathered}
$$

Definition 2.9. A continuous function $\phi(t)$ is called an upper solution of the BVP (2.22), if it satisfies

$$
\begin{gathered}
-\boldsymbol{\Phi}_{\mathfrak{t}}{ }^{\alpha-\beta} \phi(t) \geq \lambda f\left(t, I^{\beta} \phi(t), \phi(t)\right), \\
\phi(0) \leq 0, \quad \boldsymbol{\Phi}_{\mathfrak{t}}^{\gamma-\beta} \phi(1) \leq \sum_{j=1}^{p-2} a_{j} \boldsymbol{\Phi}_{\mathfrak{t}}^{\gamma-\beta} \phi\left(\xi_{j}\right) .
\end{gathered}
$$

By Lemmas 2.5 and 2.6, we have the maximal principle.

Lemma 2.10. If $1<\alpha-\beta \leq 2$ and $y \in C([0,1], R)$ satisfies

$$
y(0)=0, \quad \boldsymbol{\Phi}_{\mathbf{t}}^{\gamma-\beta} y(1)=\sum_{j=1}^{p-2} a_{j} \boldsymbol{\Phi}_{\mathbf{t}}^{\gamma-\beta} y\left(\xi_{j}\right),
$$

and $-\boldsymbol{\Phi}_{\mathbf{t}}^{\alpha-\beta} y(t) \geq 0$ for any $t \in(0,1)$, then $y(t) \geq 0$, for $t \in[0,1]$.

Set

$$
\mathcal{G}(t)=t^{\alpha-\beta-1}, \quad \mathcal{L}(t)=I^{\beta} \mathcal{G}(t)=\frac{1}{\Gamma(\beta)} \int_{0}^{t}(t-s)^{\beta-1} s^{\alpha-\beta-1} d s=\frac{\Gamma(\alpha-\beta)}{\Gamma(\alpha)} t^{\alpha-1} .
$$


To end this section, we present here two assumptions to be used throughout the rest of the paper.

(B1) $f \in C((0,1) \times(0, \infty) \times(0, \infty),[0,+\infty))$ is decreasing in $u$ and $v$, and for any $(u, v) \in$ $(0, \infty) \times(0, \infty)$,

$$
\lim _{\sigma \rightarrow+\infty} \sigma f(t, \sigma u, \sigma v)=+\infty
$$

uniformly on $t \in(0,1)$.

(B2) For any $\mu, v>0, f(t, \mu, v) \not \equiv 0$, and

$$
\int_{0}^{1}(1-s)^{\alpha-\gamma-1} f(s, \mu \mathcal{L}(s), \mu \mathcal{G}(s)) d s<+\infty
$$

\section{Main Results}

The main result is summarized in the following theorem.

Theorem 3.1. Provided that (B1) and (B2) hold, then there is a constant $\lambda^{*}>0$ such that for any $\lambda \in\left(\lambda^{*},+\infty\right)$, the problem (1.1) has at least one positive solution $x(t)$, which satisfies $x(t) \geq \mathcal{L}(t)$, $t \in[0,1]$.

Proof. Let $E=C[0,1]$; we denote a set $P$ and an operator $T_{\mathcal{\lambda}}$ in $E$ as follows:

$$
P=\left\{y \in E \text { : there exists positive number } l_{y} \text { such that } y(t) \geq l_{y} \mathcal{G}(t), t \in[0,1]\right\},
$$

$$
\left(T_{\lambda} y\right)(t)=\lambda \int_{0}^{1} K(t, s) f\left(s, I^{\beta} y(s), y(s)\right) d s, \quad \text { for any } y \in P \text {. }
$$

Clearly, $P$ is a nonempty set since $\mathcal{G}(t) \in P$. We claim that $T_{\lambda}$ is well defined and $T_{\lambda}(P) \subset P$.

In fact, for any $\rho \in P$, by the definition of $P$, there exists one positive number $l_{\rho}$ such that $\rho(t) \geq l_{\rho} \mathcal{G}(t)$ for any $t \in[0,1]$. It follows from Lemma 2.6 and (B2) that

$$
\begin{aligned}
\left(T_{\lambda} \rho\right)(t) & =\lambda \int_{0}^{1} K(t, s) f\left(s, I^{\beta} \rho(s), \rho(s)\right) d s \leq \lambda M \int_{0}^{1}(1-s)^{\alpha-\gamma-1} f\left(s, I^{\beta} \rho(s), \rho(s)\right) d s \\
& \leq \lambda M \int_{0}^{1}(1-s)^{\alpha-\gamma-1} f\left(s, l_{\rho} \mathcal{L}(s), l_{\rho} \mathcal{G}(s)\right) d s<+\infty .
\end{aligned}
$$


Setting $B=\max _{t \in[0,1]} \rho(t)>0$, from (B2), we have $f(t, B / \Gamma(\beta+1), B) \not \equiv 0$. By the continuity of $f(t, u, v)$ on $(0,1) \times(0, \infty) \times(0, \infty)$, we have $\int_{0}^{1} \mathfrak{M}(s) f(s, B / \Gamma(\beta+1), B) d s>0$. On the other hand,

$$
\begin{gathered}
I^{\beta} B=\frac{1}{\Gamma(\beta)} \int_{0}^{t}(t-s)^{\beta-1} B d s=\frac{B t^{\beta}}{\beta \Gamma(\beta)} \leq \frac{B}{\Gamma(\beta+1)}, \\
\mathfrak{M}(s)=\frac{\sum_{j=1}^{p-2} a_{j} k_{2}\left(\xi_{j}, s\right)}{1-\sum_{j=1}^{p-2} a_{j} \xi_{j}^{\alpha-\gamma-1}} \leq \frac{\sum_{j=1}^{p-2} a_{j}(1-s)^{\alpha-\gamma-1}}{\Gamma(\alpha-\beta)\left(1-\sum_{j=1}^{p-2} a_{j} \xi_{j}^{\alpha-\gamma-1}\right)} .
\end{gathered}
$$

From (3.3), one has

$$
\begin{aligned}
0 & <\int_{0}^{1} \mathfrak{M}(s) f\left(s, \frac{B}{\Gamma(\beta+1)}, B\right) d s \leq \int_{0}^{1} \mathfrak{M}(s) f\left(s, I^{\beta} B, B\right) d s \leq \int_{0}^{1} \mathfrak{M}(s) f\left(s, I^{\beta} \rho(s), \rho(s)\right) d s \\
& \leq \frac{\sum_{j=1}^{p-2} a_{j}}{\Gamma(\alpha-\beta)\left(1-\sum_{j=1}^{p-2} a_{j} \xi_{j}^{\alpha-\gamma-1}\right)} \int_{0}^{1}(1-s)^{\alpha-\gamma-1} f\left(s, I^{\beta} \rho(s), \rho(s)\right) d s<+\infty .
\end{aligned}
$$

It follows from Lemma 2.6 and (3.3) that

$$
\left(T_{\curlywedge} \rho\right)(t) \geq \lambda \mathcal{G}(t) \int_{0}^{1} \mathfrak{M}(s) f\left(s, I^{\beta} \rho(s), \rho(s)\right) d s=l_{\rho}^{\prime} \mathcal{G}(t)
$$

where

$$
l_{\rho}^{\prime}=\lambda \int_{0}^{1} \mathfrak{M}(s) f\left(s, I^{\beta} \rho(s), \rho(s)\right) d s .
$$

Using (3.3) and (3.6), we know that $T_{\lambda}$ is well defined and $T_{\lambda}(P) \subset P$.

Next we will focus on the upper and lower solutions of problem (2.22). From (B1) and (3.2), we know that the operator $T_{\lambda}$ is decreasing in $y$. Using

$$
\int_{0}^{1} K(t, s) f(s, \mathcal{L}(s), \mathcal{G}(s)) d s \geq \mathcal{G}(t) \int_{0}^{1} \mathfrak{M}(s) f(s, \mathcal{L}(s), \mathcal{G}(s)) d s, \quad \forall t \in[0,1]
$$

and letting

$$
\lambda_{1}=\frac{1}{\int_{0}^{1} \mathfrak{M}(s) f(s, \mathcal{L}(s), \mathcal{G}(s)) d s}
$$


we have

$$
\lambda_{1} \int_{0}^{1} K(t, s) f(s, \mathcal{L}(s), \mathcal{G}(s)) d s \geq \mathcal{G}(t), \quad \forall t \in[0,1] .
$$

On the other hand, letting $b(t)=\int_{0}^{1} K(t, s) f(s, \mathcal{L}(s), \mathcal{G}(s)) d s$, since $f(t, u, v)$ is decreasing with respect to $u$ and $v$, for any $\mathcal{\lambda}>\lambda_{1}$, we have

$$
\begin{aligned}
& \int_{0}^{1} K(t, s) f\left(s, \lambda I^{\beta} b(s), \lambda b(s)\right) d s \leq \int_{0}^{1} K(t, s) f\left(s, \lambda_{1} I^{\beta} b(s), \lambda_{1} b(s)\right) d s \\
& \quad \leq \int_{0}^{1} K(t, s) f(s, \mathcal{L}(s), \mathcal{G}(s)) d s \leq M \int_{0}^{1}(1-s)^{\alpha-\gamma-1} f(s, \mathcal{L}(s), \mathcal{G}(s)) d s \\
& \quad<+\infty .
\end{aligned}
$$

From (3.2), (3.3), and (B1), for all $(u, v) \in(0, \infty) \times(0, \infty)$, we have

$$
\lim _{\mu \rightarrow+\infty} \mu f(t, \mu u, \mu v)=+\infty
$$

uniformly on $t \in(0,1)$. Thus there exists large enough $\lambda^{*}>\lambda_{1}>0$, such that, for any $t \in(0,1)$,

$$
\lambda^{*} f\left(s, \lambda^{*} \mathcal{L}(s), \lambda^{*} \mathcal{G}(s)\right) \geq \frac{1}{\int_{0}^{1} \mathfrak{M}(s) d s}
$$

From Lemma 2.6, one has

$$
\begin{gathered}
\lambda^{*} \int_{0}^{1} K(t, s) f\left(s, \lambda^{*} \mathcal{L}(s), \lambda^{*} \mathcal{G}(s)\right) d s \geq \frac{\int_{0}^{1} K(t, s) d s}{\int_{0}^{1} \mathfrak{M}(s) d s} \\
\geq \frac{\int_{0}^{1} \mathcal{G}(t) \mathfrak{M}(s) d s}{\int_{0}^{1} \mathfrak{M}(s) d s}=\mathcal{G}(t), \quad \forall t \in[0,1] .
\end{gathered}
$$

Letting

$$
\begin{aligned}
& \phi(t)=\lambda^{*} \int_{0}^{1} K(t, s) f(s, \mathcal{L}(s), \mathcal{G}(s)) d s=\lambda^{*} b(t), \\
& \psi(t)=\lambda^{*} \int_{0}^{1} K(t, s) f\left(s, \lambda^{*} I^{\beta} b(s), \lambda^{*} b(s)\right) d s,
\end{aligned}
$$


and using Lemmas 2.3 and 2.7, we obtain

$$
\begin{gathered}
\phi(t)=\lambda^{*} \int_{0}^{1} K(t, s) f(s, \mathcal{L}(s), \mathcal{G}(s)) d s \geq \mathcal{G}(t), \quad t \in[0,1], \\
\phi(0)=0, \quad \boldsymbol{\Phi}_{\mathbf{t}}^{\gamma-\beta} \phi(1)=\sum_{j=1}^{p-2} a_{j} \boldsymbol{\vartheta}_{\mathbf{t}}^{\gamma-\beta} \phi\left(\xi_{j}\right), \\
\psi(t)=\lambda^{*} \int_{0}^{1} K(t, s) f\left(s, \lambda^{*} I^{\beta} b(s), \lambda^{*} b(s)\right) d s \geq \mathcal{G}(t), \quad t \in[0,1], \\
\psi(0)=0, \quad \boldsymbol{\vartheta}_{\mathbf{t}}^{\gamma-\beta} \psi(1)=\sum_{j=1}^{p-2} a_{j} \boldsymbol{\vartheta}_{\mathbf{t}}^{\gamma-\beta} \psi\left(\xi_{j}\right) .
\end{gathered}
$$

Obviously, $\phi(t), \psi(t) \in P$. By (3.16), we have

$$
\mathcal{G}(t) \leq \psi(t)=\left(T_{\lambda^{*}} \phi\right)(t), \quad \mathcal{G}(t) \leq \phi(t), \quad \forall t \in[0,1],
$$

which implies that

$$
\begin{aligned}
\psi(t) & =\left(T_{\lambda^{*}} \phi\right)(t)=\lambda^{*} \int_{0}^{1} K(t, s) f\left(s, I^{\beta} \phi(s), \phi(s)\right) d s \\
& \leq \lambda^{*} \int_{0}^{1} K(t, s) f(s, \mathcal{L}(s), \mathcal{G}(s)) d s=\phi(t), \quad \forall t \in[0,1] .
\end{aligned}
$$

Consequently, it follows from (3.17)-(3.18) that

$$
\begin{aligned}
\boldsymbol{\Phi}_{\mathfrak{t}} \psi(t)+\lambda^{*} f\left(t, I^{\beta} \psi(t), \psi(t)\right) & =\boldsymbol{\Phi}_{\mathbf{t}}\left(T_{\lambda^{*}} \phi\right)(t)+\lambda^{*} f\left(t, I^{\beta}\left(T_{\lambda^{*}} \phi\right)(t),\left(T_{\lambda^{*}} \phi\right)(t)\right) \\
& \geq \boldsymbol{\Phi}_{\mathbf{t}}\left(T_{\lambda^{*}} \phi\right)(t)+\lambda^{*} f\left(t, I^{\beta} \phi(t), \phi(t)\right) \\
& =-\lambda^{*} f\left(t, I^{\beta} \phi(t), \phi(t)\right)+\lambda^{*} f\left(t, I^{\beta} \phi(t), \phi(t)\right)=0, \\
\boldsymbol{\Phi}_{\mathbf{t}} \phi(t)+\lambda^{*} f\left(t, I^{\beta} \phi(t), \phi(t)\right) & =-\lambda^{*} f(t, \mathcal{L}(t), \mathcal{G}(t))+\lambda^{*} f\left(t, I^{\beta} \phi(t), \phi(t)\right) \\
& \leq-\lambda^{*} f(t, \mathcal{L}(t), \mathcal{G}(t))+\lambda^{*} f(t, \mathcal{L}(t), \mathcal{G}(t))=0 .
\end{aligned}
$$

From (3.16) and (3.18)-(3.20), we know that $\psi(t)$ and $\phi(t)$ are upper and lower solutions of the problem (2.22), and $\psi(t), \phi(t) \in P$.

Define the function $F$ and the operator $A_{\lambda^{*}}$ in $E$ by

$$
\begin{aligned}
& F(t, y)= \begin{cases}f\left(t, I^{\beta} \psi(t), \psi(t)\right), & y<\psi(t), \\
f\left(t, I^{\beta} y(t), y(t)\right), & \psi(t) \leq y \leq \phi(t), \\
f\left(t, I^{\beta} \phi(t), \phi(t)\right), & y>\phi(t),\end{cases} \\
& \left(A_{\lambda^{*}} y\right)(t)=\lambda^{*} \int_{0}^{1} K(t, s) F(s, y(s)) d s, \quad \forall y \in E .
\end{aligned}
$$


It follows from (B1) and (3.21) that $F:(0,1) \times[0,+\infty) \rightarrow[0,+\infty)$ is continuous. Consider the following boundary value problem:

$$
\begin{gathered}
-\boldsymbol{\Phi}_{\mathbf{t}}^{\alpha-\beta} y(t)=\lambda^{*} F(t, y), \quad t \in[0,1], \\
y(0)=0, \quad \boldsymbol{\vartheta}_{\mathbf{t}}^{\gamma-\beta} y(1)=\sum_{j=1}^{p-2} a_{j} \boldsymbol{\vartheta}_{\mathbf{t}}^{\gamma-\beta} y\left(\xi_{j}\right) .
\end{gathered}
$$

Obviously, a fixed point of the operator $A_{\lambda^{*}}$ is a solution of the BVP (3.22). For all $y \in E$, it follows from Lemma 2.6, (3.21), and $\psi(t) \geq \mathcal{G}(t)$ that

$$
\begin{aligned}
\left(A_{\lambda^{*}} y\right)(t) & \leq \lambda^{*} M \int_{0}^{1}(1-s)^{\alpha-\gamma-1} F(s, y(s)) d s \\
& \leq \lambda^{*} M \int_{0}^{1}(1-s)^{\alpha-\gamma-1} f\left(s, I^{\beta} \psi(s), \psi(s)\right) d s \\
& \leq \lambda^{*} M \int_{0}^{1}(1-s)^{\alpha-\gamma-1} f(s, \mathcal{L}(s), \mathcal{G}(s)) d s \\
& <+\infty .
\end{aligned}
$$

So $A_{\lambda^{*}}$ is bounded. From the continuity of $F(t, y)$ and $K(t, s)$, it is obviously that $A_{\lambda^{*}}: E \rightarrow E$ is continuous.

From the uniform continuity of $K(t, s)$ and the Lebesgue dominated convergence theorem, we easily get that $A_{\lambda^{*}}(\Omega)$ is equicontinuous. Thus from the Arzela-Ascoli theorem, $A_{\lambda^{*}}: E \rightarrow E$ is completely continuous. The Schauder fixed point theorem implies that $A_{\lambda^{*}}$ has at least one fixed point $w$ such that $w=A_{\lambda^{*}} w$.

Now we prove

$$
\psi(t) \leq w(t) \leq \phi(t), \quad t \in[0,1]
$$

Let $z(t)=\phi(t)-w(t), t \in[0,1]$. Since $\phi(t)$ is the upper solution of problem (2.22) and $w$ is a fixed point of $A_{\lambda^{*}}$, we have

$$
w(0)=0, \quad \boldsymbol{\nexists}_{\mathbf{t}}^{\gamma-\beta} w(1)=\sum_{j=1}^{p-2} a_{j} \boldsymbol{\Phi}_{\mathbf{t}}^{\gamma-\beta} w\left(\xi_{j}\right)
$$

From (3.17), (3.18), and the definition of $F$, we obtain

$$
\begin{gathered}
f\left(t, I^{\beta} \phi(t), \phi(t)\right) \leq F(t, y(t)) \leq f\left(t, I^{\beta} \psi(t), \psi(t)\right), \quad \forall y \in E, \\
f(t, \mathcal{L}(t), \mathcal{G}(t)) \geq f\left(t, I^{\beta} \psi(t), \psi(t)\right), \quad \forall t \in[0,1] .
\end{gathered}
$$


So

$$
f\left(t, I^{\beta} \phi(t), \phi(t)\right) \leq F(t, y(t)) \leq f(t, \mathcal{L}(t), \mathcal{G}(t)), \quad \forall y \in E
$$

From (3.18) and (3.20), one has

$$
\begin{aligned}
\boldsymbol{\Phi}_{\mathrm{t}}^{\alpha-\beta} z(t) & =\boldsymbol{\Phi}_{\mathrm{t}}{ }^{\alpha-\beta} \phi(t)-\boldsymbol{\Phi}_{\mathrm{t}}{ }^{\alpha-\beta} w(t) \\
& =-\lambda^{*} f(t, \mathcal{L}(t), \mathcal{G}(t))+\lambda^{*} F(t, w(t)) \\
& \leq 0, \quad \forall t \in[0,1] .
\end{aligned}
$$

By (3.27), (3.28), and Lemma 2.10, we get $z(t) \geq 0$ which implies that $w(t) \leq \phi(t)$ on $[0,1]$. In the same way, we have $w(t) \geq \psi(t)$ on $[0,1]$. Thus we obtain

$$
\psi(t) \leq w(t) \leq \phi(t), \quad t \in[0,1]
$$

Consequently, $F(t, w(t))=f\left(t, I^{\beta} w(t), w(t)\right), t \in[0,1]$. Then $w(t)$ is a positive solution of the problem (2.22). It thus follows from Lemma 2.7 that $x(t)=I^{\beta} w(t)$ is a positive solution of the problem (1.1).

Finally, by (3.29), we have

$$
w(t) \geq \psi(t) \geq \mathcal{G}(t)
$$

Thus,

$$
x(t)=I^{\beta} w(t)=\frac{1}{\Gamma(\beta)} \int_{0}^{t}(t-s)^{\beta-1} w(s) d s \geq \frac{1}{\Gamma(\beta)} \int_{0}^{t}(t-s)^{\beta-1} \mathcal{G}(s) d s=\mathcal{L}(t) .
$$

Corollary 3.2. Suppose that condition (B1) holds, and that for any $\mu, v>0, f(t, \mu, v) \not \equiv 0$, and

$$
\int_{0}^{1} f(s, \mu \mathcal{L}(s), \mu \mathcal{G}(s)) d s<+\infty
$$

Then there exists a constant $\lambda^{*}>0$ such that for any $\lambda \in\left(\lambda^{*},+\infty\right)$, the problem (1.1) has at least one positive solution $x(t)$, which satisfies $x(t) \geq \mathcal{L}(t), t \in[0,1]$.

We consider some special cases in which $f(t, u, v)$ has no singularity at $u, v=0$ or $t=0,1$. 
We give the following assumption.

( $\left.\mathrm{B}^{*} 1\right) f \in C((0,1) \times[0, \infty) \times[0, \infty),(0,+\infty))$ is decreasing in $u, v$.

Then, $f(t, u, v)$ is nonsingular at $u=v=0$ and for all $u, v \geq 0, f(t, u, v)>0, t \in(0,1)$, which implies that $f(t, 0,0)>0, t \in(0,1)$. Thus

$$
\lim _{\mu \rightarrow+\infty} \mu f(t, 0,0)=+\infty, \quad \text { uniformly for } t \in(0,1)
$$

naturally holds; we then have the following corollary.

Corollary 3.3. If ( $\left.\mathrm{B}^{*} 1\right)$ holds and

$\left(\mathrm{B}^{*} 2\right)$

$$
\int_{0}^{1}(1-s)^{\alpha-\gamma-1} f(s, 0,0) d s<+\infty
$$

then there exists a constant $\lambda^{*}>0$ such that for any $\lambda \in\left(\lambda^{*},+\infty\right)$, the problem (1.1) has at least one positive solution $x(t)$, which satisfies $x(t) \geq \mathcal{L}(t), t \in[0,1]$.

Proof. In the proof of Theorem 3.1, we replace the set $P$ by

$$
P_{1}=\{y \in E: y(t) \geq 0, \quad t \in[0,1]\}
$$

and the inequalities (3.18)-(3.20) by

$$
0 \leq \psi(t)=T_{\lambda} 0, \quad 0 \leq \phi(t)=T_{\lambda} \psi(t) \leq T_{\lambda} 0=\psi(t)
$$

Since $T_{\curlywedge} 0, T_{\lambda} \psi(t) \in P$, we have

$$
\begin{gathered}
\boldsymbol{\Phi}_{\mathfrak{t}}^{\alpha-\beta} T_{\lambda} 0+f\left(t, I^{\beta} T_{\lambda}, T_{\lambda} 0\right)=-f(t, 0,0)+f\left(t, I^{\beta} T_{\lambda} 0, T_{\lambda} 0\right) \leq 0, \\
\boldsymbol{\Phi}_{\mathfrak{t}}^{\alpha-\beta} T_{\lambda} \psi(t)+f\left(t, I^{\beta} T_{\lambda} \psi(t), T_{\lambda} \psi(t)\right)=-f\left(t, I^{\beta} \psi(t), \psi(t)\right)+f\left(t, I^{\beta} T_{\lambda} \psi(t), T_{\lambda} \psi(t)\right) \geq 0 \\
, t \in[0,1] .
\end{gathered}
$$

The rest of the proof is similar to that of Theorem 3.1.

If $f(t, u, v)$ is nonsingular at $u=0, v=0$ and $t=0,1$, we have the conclusion.

Corollary 3.4. If $f(t, u, v):[0,1] \times[0, \infty) \times[0, \infty) \rightarrow(0,+\infty)$ is continuous and decreasing in $u$ and $v$, the problem (1.1) has at least one positive solution $x(t)$, which satisfies $x(t) \geq \mathcal{L}(t)$, $t \in[0,1]$. 
Example 3.5. Consider the existence of positive solutions for the following eigenvalue problem of fractional differential equation:

$$
\begin{aligned}
-\boldsymbol{\vartheta}_{\mathfrak{t}}^{3 / 2} x(t) & =\frac{\lambda}{e^{t}(1-t)^{1 / 8}}\left(x^{-1 / 2}(t)+\left(\boldsymbol{\vartheta}_{\mathfrak{t}}^{1 / 8} x(t)\right)^{-1 / 8}\right), \\
\boldsymbol{\vartheta}_{\mathfrak{t}}{ }^{1 / 8} x(0) & =0, \quad \boldsymbol{\vartheta}_{\mathfrak{t}}^{3 / 8} x(1)=2 \boldsymbol{\vartheta}_{\mathfrak{t}}^{3 / 8} x\left(\frac{1}{2}\right)-\boldsymbol{\vartheta}_{\mathfrak{t}}^{3 / 8} x\left(\frac{3}{4}\right) .
\end{aligned}
$$

Let

$$
f(t, u, v)=\frac{1}{e^{t}(1-t)^{1 / 8}}\left(u^{-1 / 2}+v^{-1 / 8}\right), \quad(t, u, v) \in(0,1) \times(0,+\infty) \times(0,+\infty)
$$

Then $f \in C((0,1) \times(0,+\infty) \times(0,+\infty),(0,+\infty))$ is decreasing in $u$ and $v$, and for any $(u, v) \in$ $(0, \infty) \times(0, \infty)$,

$$
\lim _{\sigma \rightarrow+\infty} \sigma f(t, \sigma u, \sigma v)=\lim _{\sigma \rightarrow+\infty} \frac{\sigma^{1 / 2} u^{-1 / 2}+\sigma^{7 / 8} v^{-1 / 8}}{e^{t}(1-t)^{1 / 8}}=+\infty
$$

uniformly on $t \in(0,1)$. Thus (B1) holds.

On the other hand, for any $\mu, v>0$ and $t \in(0,1)$,

$$
\begin{aligned}
& f(t, \mu, v)=\frac{1}{e^{t}(1-t)^{1 / 8}}\left(\mu^{-1 / 2}+v^{-1 / 8}\right) \not \equiv 0 \\
& \mathcal{L}(t)=\int_{0}^{t} \frac{(t-s)^{-7 / 8} s^{3 / 8}}{\Gamma(1 / 8)} d s=\frac{\Gamma(11 / 8)}{\Gamma(3 / 2)} t^{1 / 2}
\end{aligned}
$$

thus we have

$$
\begin{aligned}
\int_{0}^{1}(1-s)^{\alpha-\gamma-1} f(s, \mu \mathcal{L}(s), \mu \mathcal{G}(s)) d s= & \int_{0}^{1}(1-s)^{1 / 8}\left[\frac { 1 } { e ^ { s } ( 1 - s ) ^ { 1 / 8 } } \left(\mu^{-1 / 2} \mathcal{L}^{-1 / 2}(s)\right.\right. \\
\left.\left.+\mu^{-1 / 8} \mathcal{G}^{-1 / 8}(s)\right)\right] & \\
\leq & \int_{0}^{1}\left[\mu^{-1 / 2}\left(\frac{\Gamma(11 / 8)}{\Gamma(3 / 2)} t^{1 / 2}\right)^{-1 / 2}+\mu^{-1 / 8} s^{-3 / 64}\right] d s \\
& =\int_{0}^{1}\left[\mu^{-1 / 2}\left(\frac{\Gamma(11 / 8)}{\Gamma(3 / 2)} t^{-1 / 4}\right)+\mu^{-1 / 8} s^{-3 / 64}\right] d s<+\infty,
\end{aligned}
$$


which implies that (B2) holds. From Theorem 3.1, there is a constant $\lambda^{*}>0$ such that for any $\lambda \in\left(\lambda^{*},+\infty\right)$ the problem (3.38) has at least one positive solution $x(t)$ and

$$
x(t) \geq \mathcal{L}(t)=\frac{\Gamma(11 / 8)}{\Gamma(3 / 2)} t^{1 / 2} \approx 1.003 t^{1 / 2}, \quad t \in[0,1] .
$$

\section{Acknowledgments}

This work is supported financially by the National Natural Science Foundation of China (11071141, 11126231) and the Natural Science Foundation of Shandong Province of China (ZR2010AM017).

\section{References}

[1] I. Podlubny, Fractional Differential Equations, vol. 198 of Mathematics in Science and Engineering, Academic Press, San Diego, Calif, USA, 1999.

[2] S. G. Samko, A. A. Kilbas, and O. I. Marichev, Fractional Integrals and Derivatives, Theory and Applications, Gordon and Breach, Yverdon, Switzerland, 1993.

[3] K. S. Miller and B. Ross, An Introduction to the Fractional Calculus and Fractional Differential Equations, John Wiley \& Sons, New York, NY, USA, 1993.

[4] A. A. Kilbas and J. J. Trujillo, "Differential equations of fractional order: methods, results and problems. I," Applicable Analysis, vol. 78, no. 1-2, pp. 153-192, 2001.

[5] A. A. Kilbas and J. J. Trujillo, "Differential equations of fractional order: methods, results and problems. II," Applicable Analysis, vol. 81, no. 2, pp. 435-493, 2002.

[6] A. A. Kilbas, H. M. Srivastava, and J. J. Trujillo, Theory and Applications of Fractional Differential Equations, vol. 204 of North-Holland Mathematics Studies, Elsevier Science B.V., Amsterdam, The Netherlands, 2006.

[7] T. G. Bhaskar, V. Lakshmikantham, and S. Leela, "Fractional differential equations with a Krasnoselskii-Krein type condition," Nonlinear Analysis, vol. 3, no. 4, pp. 734-737, 2009.

[8] M. ur Rehman and R. A. Khan, "Existence and uniqueness of solutions for multi-point boundary value problems for fractional differential equations," Applied Mathematics Letters, vol. 23, no. 9, pp. 1038-1044, 2010.

[9] X. Zhang, L. Liu, and Y. Wu, "Multiple positive solutions of a singular fractional differential equation with negatively perturbed term," Mathematical and Computer Modelling, vol. 55, no. 3-4, pp. 1263-1274, 2012.

[10] D. Jiang and C. Yuan, "The positive properties of the Green function for Dirichlet-type boundary value problems of nonlinear fractional differential equations and its application," Nonlinear Analysis: Theory, Methods E Applications A, vol. 72, no. 2, pp. 710-719, 2010.

[11] X. Zhang and Y. Han, "Existence and uniqueness of positive solutions for higher order nonlocal fractional differential equations," Applied Mathematics Letters, vol. 25, no. 3, pp. 555-560, 2012.

[12] C. S. Goodrich, "Existence of a positive solution to a class of fractional differential equations," Applied Mathematics Letters, vol. 23, no. 9, pp. 1050-1055, 2010.

[13] C. S. Goodrich, "Existence of a positive solution to systems of differential equations of fractional order," Computers \& Mathematics with Applications, vol. 62, no. 3, pp. 1251-1268, 2011.

[14] M. El-Shahed and J. J. Nieto, "Nontrivial solutions for a nonlinear multi-point boundary value problem of fractional order," Computers \& Mathematics with Applications, vol. 59, no. 11, pp. 3438-3443, 2010.

[15] X. Zhang, L. Liu, and Y. Wu, "The eigenvalue problem for a singular higher order fractional differential equation involving fractional derivatives," Applied Mathematics and Computation, vol. 218, no. 17, pp. 8526-8536, 2012.

[16] Y. Wang, L. Liu, and Y. H. Wu, "Positive solutions for a nonlocal fractional differential equation," Nonlinear Analysis, vol. 74, no. 11, pp. 3599-3605, 2011.

[17] Y. Wang, L. Liu, and Y. H. Wu, "Positive solutions for a class of fractional boundary value problem with changing sign nonlinearity," Nonlinear Analysis, vol. 74, no. 17, pp. 6434-6441, 2011. 


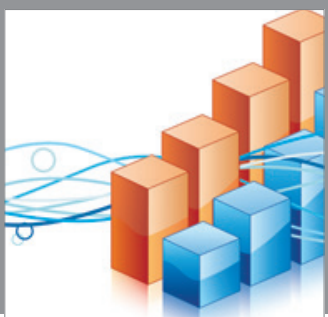

Advances in

Operations Research

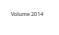

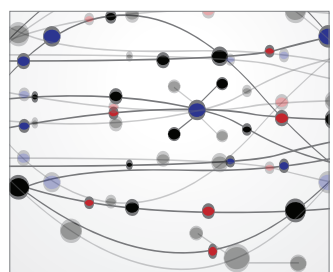

\section{The Scientific} World Journal
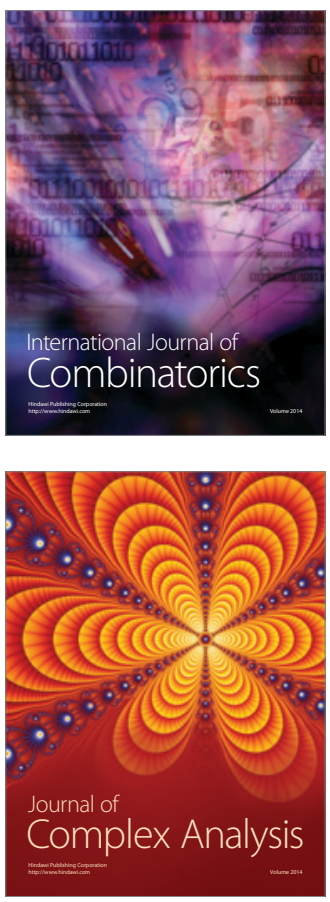

International Journal of

Mathematics and

Mathematical

Sciences
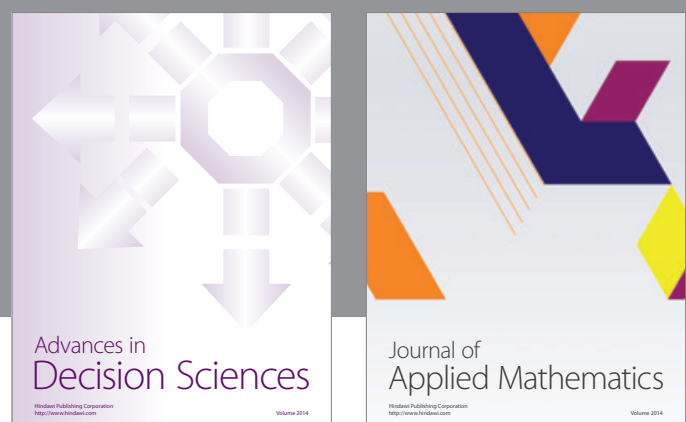

Journal of

Applied Mathematics
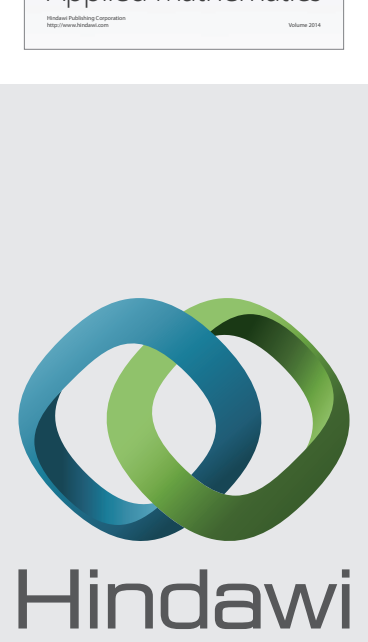

Submit your manuscripts at http://www.hindawi.com
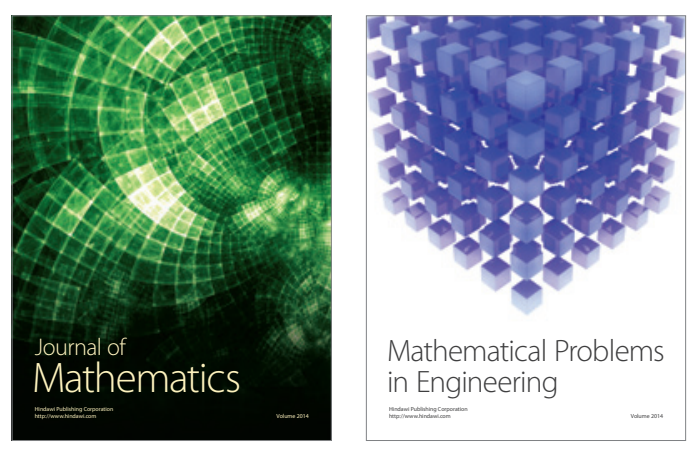

Mathematical Problems in Engineering
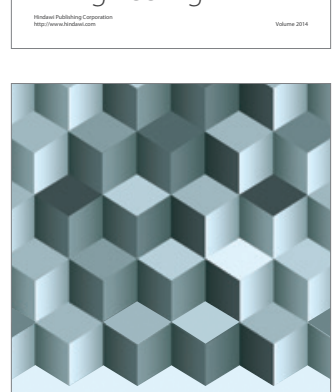

Journal of

Function Spaces
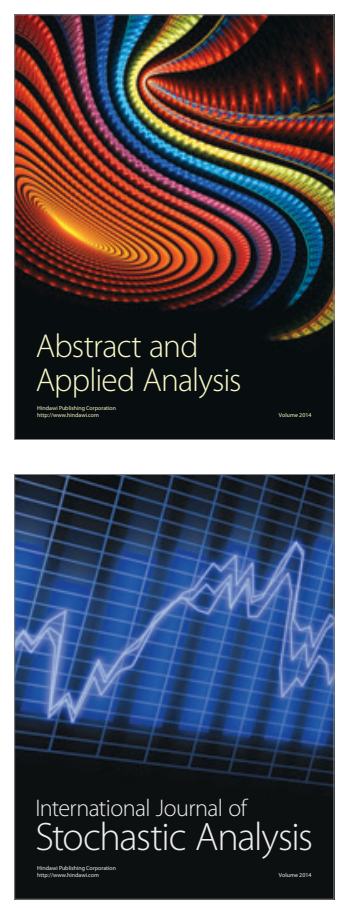

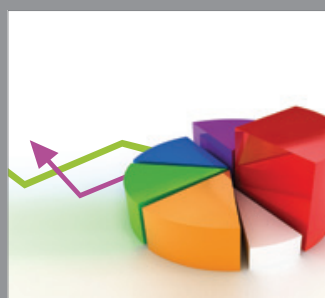

ournal of

Probability and Statistics

Promensencen
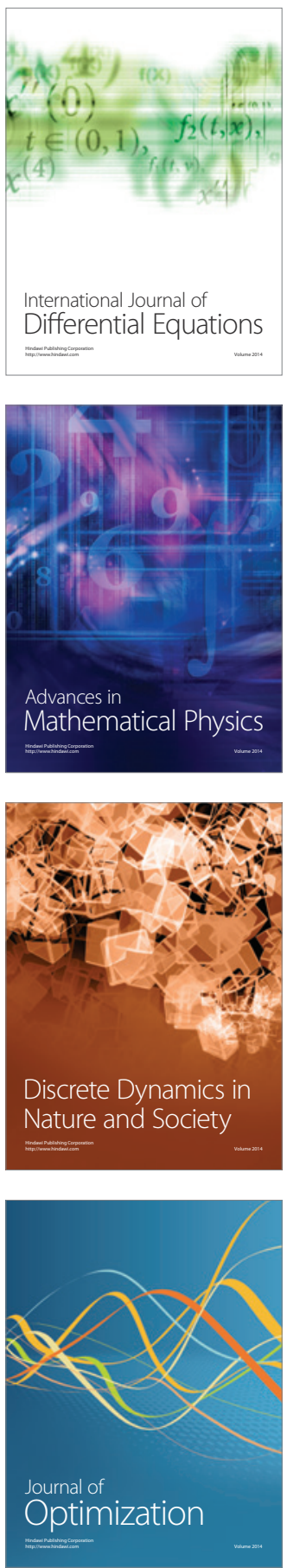